

\title{
A lower bound on the essential dimension of simple algebras
}

\author{
Alexander S. Merkurjev
}

Let $p$ be a prime integer and $F$ a field of characteristic different from $p$. We prove that the essential $p$-dimension $\operatorname{ed}_{p}\left(\operatorname{CSA}\left(p^{r}\right)\right)$ of the class $\operatorname{CSA}\left(p^{r}\right)$ of central simple algebras of degree $p^{r}$ is at least $(r-1) p^{r}+1$. The integer $\operatorname{ed}_{p}\left(\operatorname{CSA}\left(p^{r}\right)\right)$ measures complexity of the class of central simple algebras of degree $p^{r}$ over field extensions of $F$.

\section{Introduction}

The essential dimension of an algebraic structure is a numerical invariant that measures its complexity. Informally, the essential dimension of an algebraic structure over a field $F$ is the smallest number of algebraically independent parameters required to define the structure over a field extension of $F$ [Berhuy and Favi 2003; Merkurjev 2009].

Let $\mathscr{F}:$ Fields $/ F \rightarrow$ Sets be a functor (an algebraic structure) from the category Fields / $F$ of field extensions of $F$ and field homomorphisms over $F$ to the category of sets. Let $K \in$ Fields $/ F, \alpha \in \mathscr{F}(K)$, and $K_{0}$ be a subfield of $K$ over $F$. We say that $\alpha$ is defined over $K_{0}$ (and $K_{0}$ is called a field of definition of $\alpha$ ) if there exists an element $\alpha_{0} \in \mathscr{F}\left(K_{0}\right)$ such that the image $\left(\alpha_{0}\right)_{K}$ of $\alpha_{0}$ under the map $\mathscr{F}\left(K_{0}\right) \rightarrow \mathscr{F}(K)$ coincides with $\alpha$. The essential dimension of $\alpha$, denoted ed ${ }^{\mathscr{F}}(\alpha)$, is the least transcendence degree tr. $\operatorname{deg}_{F}\left(K_{0}\right)$ over all fields of definition $K_{0}$ of $\alpha$. The essential dimension of the functor $\mathscr{F}$ is

$$
\operatorname{ed}(\mathscr{F})=\sup \left\{\operatorname{ed}^{\mathscr{F}}(\alpha)\right\},
$$

where the supremum is taken over fields $K \in$ Fields $/ F$ and all $\alpha \in \mathscr{F}(K)$.

Let $p$ be a prime integer and $\alpha \in \mathscr{F}(K)$. The essential $p$-dimension $\operatorname{ed}_{p}^{\mathscr{F}}(\alpha)$ of $\alpha$ is the minimum of ed $^{\mathscr{F}}\left(\alpha_{K^{\prime}}\right)$ over all finite field extensions $K^{\prime} / K$ of degree prime to $p$. The essential $p$-dimension $\operatorname{ed}_{p}(\mathscr{F})$ of $\mathscr{F}$ is the supremum of $\operatorname{ed}_{p}^{\mathscr{F}}(\alpha)$ over all

The work has been supported by the NSF grant DMS \#0652316.

MSC2000: primary 16K50; secondary 14L30, $20 \mathrm{G} 15$.

Keywords: essential dimension, Brauer group, algebraic tori. 
fields $K \in$ Fields $/ F$ and all $\alpha \in \mathscr{F}(K)$ [Reichstein and Youssin 2000, §6]. Clearly, $\operatorname{ed}^{\mathscr{F}}(\alpha) \geq \operatorname{ed}_{p}^{\mathscr{F}}(\alpha)$ and $\operatorname{ed}(\mathscr{F}) \geq \operatorname{ed}_{p}(\mathscr{F})$ for all $p$.

Let $\operatorname{CSA}(n)$ be the functor taking a field extension $K / F$ to the set of isomorphism classes $\operatorname{CSA}_{K}(n)$ of central simple $K$-algebras of degree $n$. Let $p$ be a prime integer and let $p^{r}$ be the highest power of $p$ dividing $n$. Then $\operatorname{ed}_{p}(C S A(n))=$ $\operatorname{ed}_{p}\left(C S A\left(p^{r}\right)\right)$ [Reichstein and Youssin 2000, Lemma 8.5.5]. Every central simple algebra of degree $p$ is cyclic over a finite field extension of degree prime to $p$, and hence $\operatorname{ed}_{p}(C S A(p))=2$ [Reichstein and Youssin 2000, Lemma 8.5.7]. It was proven in [Merkurjev 2010] that $\operatorname{ed}_{p}\left(C S A\left(p^{2}\right)\right)=p^{2}+1$ and in general, $2 p^{2 r-2}-p^{r}+1 \geq \operatorname{ed}_{p}\left(C S A\left(p^{r}\right)\right) \geq 2 r$ for all $r \geq 2$ [Meyer and Reichstein 2009b, Theorem 1; Reichstein and Youssin 2000, Theorem 8.6].

We improve the lower bound for $\operatorname{ed}_{p}\left(\operatorname{CSA}\left(p^{r}\right)\right)$ as follows:

Theorem 6.1. Let $F$ be a field and $p$ a prime integer different from $\operatorname{char}(F)$. Then

$$
\operatorname{ed}_{p}\left(\operatorname{CSA}\left(p^{r}\right)\right) \geq(r-1) p^{r}+1 .
$$

Let $G$ be an algebraic group over $F$. The essential dimension $\operatorname{ed}(G)$ (resp. essential $p$-dimension $\operatorname{ed}_{p}(G)$ ) of $G$ is the essential dimension (resp. essential $p$ dimension) of the functor $G$-torsors taking a field $K$ to the set of isomorphism classes of all $G$-torsors (principal homogeneous $G$-spaces) over $K$.

If $G=\mathbf{P G L}(n)$ is the projective linear group over $F$, the functor $G$-torsors is isomorphic to the functor $\operatorname{CSA}(n)$. Therefore, the theorem yields the following lower bound for the essential dimension of $\operatorname{PGL}\left(p^{r}\right)$ :

$$
\operatorname{ed}\left(\operatorname{PGL}\left(p^{r}\right)\right) \geq \operatorname{ed}_{p}\left(\operatorname{PGL}\left(p^{r}\right)\right) \geq(r-1) p^{r}+1 .
$$

\section{Preliminaries}

Characters. Let $F$ be a field, let $F_{\text {sep }}$ be a separable closure of $F$, and let

$$
\Gamma=\operatorname{Gal}\left(F_{\text {sep }} / F\right)
$$

be the absolute Galois group of $F$. For a $\Gamma$-module $M$, we write $H^{n}(F, M)$ for the cohomology group $H^{n}(\Gamma, M)$.

The character group $\mathrm{Ch}(F)$ of $F$ is defined as

$$
\operatorname{Hom}_{\mathrm{cont}}(\Gamma, \mathbb{Q} / \mathbb{Z})=H^{1}(F, \mathbb{Q} / \mathbb{Z}) \simeq H^{2}(F, \mathbb{Z}) .
$$

For a character $\chi \in \mathrm{Ch}(F)$, set $F(\chi)=\left(F_{\text {sep }}\right)^{\operatorname{Ker}(\chi)}$. Then $F(\chi) / F$ is a cyclic field extension of degree ord $(\chi)$. If $\Phi \subset \mathrm{Ch}(F)$ is a finite subgroup, we set

$$
F(\Phi)=\left(F_{\text {sep }}\right)^{\cap \operatorname{Ker}(\chi)},
$$


where the intersection is taken over all $\chi \in \Phi$. The Galois group $G=\operatorname{Gal}(F(\Phi) / F)$ is abelian and $\Phi$ is canonically isomorphic to the character group $\operatorname{Hom}(G, \mathbb{Q} / \mathbb{Z})$ of $G$.

If $F^{\prime} \subset F$ is a subfield and $\chi \in \mathrm{Ch}\left(F^{\prime}\right)$, we write $\chi_{F}$ for the image of $\chi$ under the natural map $\mathrm{Ch}\left(F^{\prime}\right) \rightarrow \mathrm{Ch}(F)$ and $F(\chi)$ for $F\left(\chi_{F}\right)$. If $\Phi \subset \mathrm{Ch}(F)$ is a finite subgroup, then the character $\chi_{F(\Phi)}$ is trivial if and only if $\chi \in \Phi$.

Lemma 2.1. Let $\Phi, \Phi^{\prime} \subset \mathrm{Ch}(F)$ be two finite subgroups. Suppose that for a field extension $K / F$, we have $\Phi_{K}=\Phi_{K}^{\prime}$ in $\mathrm{Ch}(K)$. Then there is a finite subextension $K^{\prime} / F$ in $K / F$ such that $\Phi_{K^{\prime}}=\Phi_{K^{\prime}}^{\prime}$ in $\mathrm{Ch}\left(K^{\prime}\right)$.

Proof. Choose a set of characters $\left\{\chi_{1}, \ldots, \chi_{m}\right\}$ generating $\Phi$ and a set of characters $\left\{\chi_{1}^{\prime}, \ldots, \chi_{m}^{\prime}\right\}$ generating $\Phi^{\prime}$ such that $\left(\chi_{i}\right)_{K}=\left(\chi_{i}^{\prime}\right)_{K}$ for all $i$. Let $\eta_{i}=\chi_{i}-\chi_{i}^{\prime}$. Since all $\eta_{i}$ vanish over $K$, the finite field extension $K^{\prime}:=F\left(\eta_{1}, \ldots, \eta_{m}\right)$ of $F$ can be viewed as a subextension in $K / F$. Now $\Phi_{K^{\prime}}=\Phi_{K^{\prime}}^{\prime}$ since $\left(\chi_{i}\right)_{K^{\prime}}=\left(\chi_{i}^{\prime}\right)_{K^{\prime}}$.

Brauer groups. We write $\operatorname{Br}(F)$ for the Brauer group $H^{2}\left(F, F_{\text {sep }}^{\times}\right)$of a field $F$. If $a \in \operatorname{Br}(F)$ and $K / F$ is a field extension, then we write $a_{K}$ for the image of $a$ under the natural homomorphism $\operatorname{Br}(F) \rightarrow \operatorname{Br}(K)$. We write $\operatorname{Br}(K / F)$ for the relative Brauer group $\operatorname{Ker}(\operatorname{Br}(F) \rightarrow \operatorname{Br}(K))$. We say that $K$ is a splitting field of $a$ if $a_{K}=0$, that is, $a \in \operatorname{Br}(K / F)$. The index $\operatorname{ind}(a)$ of $a$ is the smallest degree of a splitting field of $a$.

The cup product

$$
\mathrm{Ch}(F) \otimes F^{\times}=H^{2}(F, \mathbb{Z}) \otimes H^{0}\left(F, F_{\text {sep }}^{\times}\right) \rightarrow H^{2}\left(F, F_{\text {sep }}^{\times}\right)=\operatorname{Br}(F)
$$

takes $\chi \otimes a$ to the class $\chi \cup(a)$ in $\operatorname{Br}(F)$ that is split by $F(\chi)$.

For a finite subgroup $\Phi \subset \mathrm{Ch}(F)$, write $\operatorname{Br}_{\operatorname{dec}}(F(\Phi) / F)$ for the subgroup of decomposable elements in $\operatorname{Br}(F(\Phi) / F)$ generated by the elements $\chi \cup(a)$ for all $\chi \in \Phi$ and $a \in F^{\times}$. The indecomposable relative Brauer group $\mathrm{Br}_{\text {ind }}(F(\Phi) / F)$ is the factor group $\operatorname{Br}(F(\Phi) / F) / \operatorname{Br}_{\mathrm{dec}}(F(\Phi) / F)$.

Complete fields. Let $E$ be a complete field with respect to a discrete valuation $v$, and let $K$ be its residue field.

Let $p$ be a prime integer different from $\operatorname{char}(K)$. There is a natural injective homomorphism $\mathrm{Ch}(K)\{p\} \rightarrow \mathrm{Ch}(E)\{p\}$ of the $p$-primary components of the character groups that identifies $\mathrm{Ch}(K)\{p\}$ with the character group of an unramified field extension of $E$. For a character $\chi \in \operatorname{Ch}(K)\{p\}$, we write $\hat{\chi}$ for the corresponding character in $\mathrm{Ch}(E)\{p\}$.

By [Garibaldi et al. 2003, §7.9], there is an exact sequence

$$
0 \rightarrow \operatorname{Br}(K)\{p\} \stackrel{i}{\rightarrow} \operatorname{Br}(E)\{p\} \stackrel{\partial_{v}}{\rightarrow} \mathrm{Ch}(K)\{p\} \rightarrow 0 .
$$


If $a \in \operatorname{Br}(K)\{p\}$, we write $\hat{a}$ for the element $i(a)$ in $\operatorname{Br}(E)\{p\}$. For example, if $a=\chi \cup(\bar{u})$ for some $\chi \in \operatorname{Ch}(K)\{p\}$ and a unit $u \in E$, then $\hat{a}=\hat{\chi} \cup(u)$.

Proposition 2.2 [Tignol 1978, Proposition 2.4; Jacob and Wadsworth 1990, Theorem 5.15(a); Garibaldi et al. 2003, Proposition 8.2]. Let E be a complete field with respect to a discrete valuation $v$, and let $K$ be its residue field of characteristic different from $p$. Then:

(i) $\operatorname{ind}(\hat{a})=\operatorname{ind}(a)$ for any $a \in \operatorname{Br}(K)\{p\}$.

(ii) Let $b=\hat{a}+(\hat{\chi} \cup(x))$ for an element $a \in \operatorname{Br}(K)\{p\}, \chi \in \operatorname{Ch}(K)\{p\}$ and $x \in E^{\times}$. Then $\partial_{v}(b)=v(x) \chi$. Also, if $v(x)$ is not divisible by $p$, we have

$$
\operatorname{ind}(b)=\operatorname{ind}\left(a_{K(\chi)}\right) \cdot \operatorname{ord}(\chi) .
$$

(iii) Let $E^{\prime} / E$ be a finite field extension and $v^{\prime}$ the discrete valuation on $E^{\prime}$ extending $v$ with residue field $K^{\prime}$. Then for any $b \in \operatorname{Br}(E)\{p\}$, we have

$$
\partial_{v^{\prime}}\left(b_{E^{\prime}}\right)=e \cdot \partial_{v}(b)_{K^{\prime}},
$$

where $e$ is the ramification index of $E^{\prime} / E$.

The choice of a prime element $\pi$ in $E$ provides us with a splitting of the sequence (2-1) by sending a character $\chi$ to the class $\hat{\chi} \cup(\pi)$ in $\operatorname{Br}(E)\{p\}$. Thus, any $b \in$ $\operatorname{Br}(E)\{p\}$ can be written in the form

$$
b=\hat{a}+(\hat{\chi} \cup(\pi)),
$$

for $\chi=\partial_{v}(b)$ and a unique $a \in \operatorname{Br}(K)\{p\}$.

The homomorphism

$$
s_{\pi}: \operatorname{Br}(E)\{p\} \rightarrow \operatorname{Br}(K)\{p\},
$$

defined by $s_{\pi}(b)=a$, where $a$ is given by (2-2), is called a specialization map. For example, $s_{\pi}(\hat{a})=a$ for any $a \in \operatorname{Br}(K)\{p\}$ and $s_{\pi}(\hat{\chi} \cup(x))=\chi \cup(\bar{u})$, where $\chi \in \operatorname{Ch}(K)\{p\}, x \in E^{\times}$and $u$ is the unit in $E$ such that $x=u \pi^{v(x)}$.

If $v$ is trivial on a subfield $F \subset E$ and $\Phi \subset \mathrm{Ch}(F)\{p\}$ a finite subgroup, then

$$
s_{\pi}\left(\operatorname{Br}_{\mathrm{dec}}(E(\Phi) / E)\right) \subset \mathrm{Br}_{\mathrm{dec}}(K(\Phi) / K) .
$$

We shall need the following technical lemma. For an abelian group $A$ we write ${ }_{p} A$ for the subgroup of all elements in $A$ of exponent dividing $p$.

Lemma 2.3. Let $(E, v)$ be a complete discrete valued field with the residue field $K$ of characteristic different from $p$ containing a primitive $p^{2}$-th root of unity. Let $\eta \in \mathrm{Ch}(E)$ be a character of order $p^{2}$ such that $p \cdot \eta$ is unramified, that is, $p \cdot \eta=\hat{v}$ for some $v \in \mathrm{Ch}(K)$ of order $p$. Let $\chi \in{ }_{p} \mathrm{Ch}(K)$ be a character linearly independent from $v$. Let $a \in \operatorname{Br}(K)$ and set $b=\hat{a}+(\hat{\chi} \cup(x)) \in \operatorname{Br}(E)$, where $x \in E^{\times}$is an element such that $v(x)$ is not divisible by $p$. Then: 
(i) If $\eta$ is unramified, that is, $\eta=\hat{\mu}$ for some $\mu \in \mathrm{Ch}(K)$ of order $p^{2}$, then $\operatorname{ind}\left(b_{E(\eta)}\right)=p \cdot \operatorname{ind}\left(a_{K(\mu, \chi)}\right)$.

(ii) If $\eta$ is ramified, then there exists a unit $u \in E^{\times}$such that $K(v)=K\left(\bar{u}^{1 / p}\right)$ and $\operatorname{ind}\left(b_{E(\eta)}\right)=\operatorname{ind}\left(a-\left(\chi \cup\left(\bar{u}^{1 / p}\right)\right)\right)_{K(v)}$.

Proof. (i) If $\eta=\hat{\mu}$ for some $\mu \in \mathrm{Ch}(K)$, then $K(\mu)$ is the residue field of $E(\eta)$ and we have

$$
b_{E(\eta)}=\hat{a}_{K(\mu)}+\left(\hat{\chi}_{K(\mu)} \cup(x)\right) \text {. }
$$

Since $\chi$ and $v$ are linearly independent, the character $\chi_{K(\mu)}$ is nontrivial. The first statement follows from Proposition 2.2(ii).

(ii) Since $p \cdot \eta$ is unramified, the ramification index of $E(\eta) / E$ is equal to $p$, and hence $E(\eta)=E\left(\left(u x^{p}\right)^{1 / p^{2}}\right)$ for some unit $u \in E$. Note that $K(v)=K\left(\bar{u}^{1 / p}\right)$ is the residue field of $E(\eta)$. Since $u^{1 / p} x$ is a $p$ th power in $E(\eta)$, the class

$$
\left.b_{E(\eta)}=\hat{a}_{K(v)}-\left(\hat{\chi}_{K(v)} \cup\left(u^{1 / p}\right)\right)=\hat{a}_{K(v)}-\left(\chi_{K(v)} \widehat{\cup\left(\bar{u}^{1}\right.} 1 / p\right)\right)
$$

is unramified. It follows from Proposition 2.2(i) that the elements $b_{E(\eta)} \operatorname{in} \operatorname{Br}(E(\eta))$ and $a_{K(v)}-\left(\chi_{K(v)} \cup\left(\bar{u}^{1 / p}\right)\right)$ in $\operatorname{Br}(K(v))$ have the same indices.

\section{Brauer group and algebraic tori}

Torsors. Let $G$ be an algebraic group over $F$ and let $K / F$ be a field extension. The set of isomorphism classes of $G$-torsors (principal homogeneous spaces) over $K$ is bijective to $H^{1}(K, G)$ [Serre 1997].

Example 3.1. Let $A$ be a central simple $F$-algebra of degree $n$ and $G=\operatorname{Aut}(A)$. Then $H^{1}(K, G)$ is the set of isomorphism classes of central simple $K$-algebras of degree $n$, or equivalently, the set of elements in $\operatorname{Br}(K)$ of index dividing $n$. If $A=M_{n}(F)$ is the split algebra, then $G=\operatorname{PGL}(n)$.

Example 3.2. Let $L$ be an étale $F$-algebra of dimension $n$. Consider the algebraic torus $U=R_{L / F}\left(\mathbb{G}_{m, L}\right) / \mathbb{G}_{m}$ over $F$. The exact sequence

$$
1 \rightarrow \mathbb{G}_{m} \rightarrow R_{L / F}\left(\mathbb{G}_{m, L}\right) \rightarrow U \rightarrow 1
$$

and Hilbert Theorem 90 yield an isomorphism $\theta: H^{1}(F, U) \stackrel{\sim}{\rightarrow} \operatorname{Br}(L / F)$. Note that if $L$ is a subalgebra of a central simple $F$-algebra $A$ of degree $n$, then $U$ is a maximal torus in the group $\operatorname{Aut}(A)$.

Let $\alpha: G \rightarrow \mathbf{G L}(W)$ be a finite dimensional representation over $F$. Suppose that $\alpha$ is generically free, that is, there is a nonempty open subset $W^{\prime} \subset W$ and a $G$-torsor $\beta: W^{\prime} \rightarrow X$ for a variety $X$ over $F$. The torsor $\beta$ is versal, that is, every $G$-torsor over a field extension $K / F$ is the pull-back of $\beta$ with respect to a $K$-point of $X$. The generic fiber of $\beta$ is called a generic $G$-torsor. It is a torsor over the function field $F(X)$ [Garibaldi et al. 2003; Reichstein 2000]. 
Example 3.3. Let $S$ be an algebraic torus over $F$. We embed $S$ into the quasitrivial torus $P=R_{L / F}\left(\mathbb{G}_{m, L}\right)$, where $L$ is an étale $F$-algebra [Colliot-Thélène and Sansuc 1977]. Then $S$ acts on the vector space $L$ by multiplication, so that the action on the open subset $P$ is regular. If $T$ is the factor torus $P / S$, then the $S$-torsor $P \rightarrow T$ is versal.

The tori $P^{\Phi}, S^{\Phi}, T^{\Phi}, U^{\Phi}$ and $V^{\Phi}$. Let $F$ be a field, $\Phi$ be a subgroup of ${ }_{p} \operatorname{Ch}(F)$ of rank $r$, and $L=F(\Phi)$. Let $G=\operatorname{Gal}(L / F)$. Choose a basis $\chi_{1}, \chi_{2}, \ldots, \chi_{r}$ for $\Phi$. We can view each $\chi_{i}$ as a character of $G$, that is, as a homomorphism $\chi_{i}: G \rightarrow \mathbb{Q} / \mathbb{Z}$. Let $\sigma_{1}, \sigma_{2}, \ldots, \sigma_{r}$ be the dual basis for $G$, that is,

$$
\chi_{i}\left(\sigma_{j}\right)= \begin{cases}(1 / p)+\mathbb{Z} & \text { if } i=j, \\ 0 & \text { otherwise. }\end{cases}
$$

Let $R$ be the group ring $\mathbb{Z}[G]$. Consider the surjective homomorphism of $G$ modules $k: R^{r} \rightarrow R$ taking the $i$ th basis element $e_{i}$ of $R^{r}$ to $\sigma_{i}-1$. The image of $k$ is the augmentation ideal $I=\operatorname{Ker}(\varepsilon)$ in $R$, where $\varepsilon: R \rightarrow \mathbb{Z}$ is defined by $\varepsilon(\rho)=1$ for all $\rho \in G$.

Write $N_{i}=1+\sigma_{i}+\sigma_{i}^{2}+\cdots+\sigma_{i}^{p-1} \in R$.

Set $N:=\operatorname{Ker}(k)$. Consider the following elements in $N$ :

$$
e_{i j}:=\left(\sigma_{i}-1\right) e_{j}-\left(\sigma_{j}-1\right) e_{i} \quad \text { and } \quad f_{i}=N_{i} e_{i}, \quad i, j=1, \ldots r .
$$

Lemma 3.4. The G-module $N$ is generated by $e_{i j}$ and $f_{i}$.

Proof. Let $\bar{R}=\mathbb{Z}\left[t_{1}, \ldots, t_{r}\right]$ be the polynomial ring. Acyclicity of the Koszul complex for the homomorphism $\bar{k}:(\bar{R})^{r} \rightarrow \bar{R}$, taking the $i$ th basis element $\bar{e}_{i}$ to $t_{i}-1$ [Matsumura 1980, Theorem 43] implies that $\operatorname{Ker}(\bar{k})$ is generated by $\bar{e}_{i j}:=$ $\left(t_{i}-1\right) \bar{e}_{j}-\left(t_{j}-1\right) \bar{e}_{i}$.

The kernel $J$ of the surjective homomorphism $\bar{R} \rightarrow R$, taking $t_{i}$ to $\sigma_{i}$, is generated by $t_{i}^{p}-1$.

Let $x:=\sum x_{i} e_{i} \in \operatorname{Ker}(k)$. Lift every $x_{i}$ to a polynomial $\bar{x}_{i} \in \bar{R}$ and consider $\bar{x}:=\sum \bar{x}_{i} \bar{e}_{i} \in(\bar{R})^{r}$. We have $\bar{k}(\bar{x}) \in J$, and hence

$$
\bar{k}(\bar{x})=\sum\left(t_{i}-1\right) \bar{x}_{i}=\sum\left(t_{i}^{p}-1\right) h_{i}=\sum\left(t_{i}-1\right) \bar{N}_{i} h_{i},
$$

for some polynomials $h_{i} \in \bar{R}$, where $\bar{N}_{i}=1+t_{i}+t_{i}^{2}+\cdots+t_{i}^{p-1} \in R$. Hence the element $\sum\left(\bar{x}_{i}-h_{i} \bar{N}_{i}\right) \bar{e}_{i}$ belongs to the kernel of $\bar{k}$ and therefore is a linear combination of $\bar{e}_{i j}$. It follows that $\bar{x}$ is a linear combination of $\bar{e}_{i j}$ and $\bar{N}_{i} \bar{e}_{i}$, and hence $x$ is a linear combination of $e_{i j}$ and $f_{i}$.

Let $\varepsilon_{i}: R^{r} \rightarrow \mathbb{Z}$ be the $i$ th projection followed by the augmentation map $\varepsilon$. It follows from Lemma 3.4 that $\varepsilon_{i}(N)=p \mathbb{Z}$ for every $i$. Moreover, the $G$ homomorphism

$$
l: N \rightarrow \mathbb{Z}^{r}, \quad m \mapsto\left(\varepsilon_{1}(m) / p, \ldots, \varepsilon_{r}(m) / p\right)
$$


is surjective. Set $M=\operatorname{Ker}(l)$ and $Q=R^{r} / M$.

Lemma 3.5. The $G$-module $M$ is generated by $e_{i j}$.

Proof. Let $M^{\prime}$ be the submodule of $N$ generated by $e_{i j}$. Clearly, $M^{\prime} \subset M$. Note also that $\left(\sigma_{j}-1\right) f_{i}=N_{i} e_{i j} \in M^{\prime}$, and hence $I f_{i} \subset M^{\prime}$.

Suppose that $m \in M$. By Lemma 3.4, modifying $m$ by an element in $M^{\prime}$, we can assume that $m=\sum_{i=1}^{r} x_{i} f_{i}$ for some $x_{i} \in R$. Since $l(m)=0$, we have $\varepsilon\left(x_{i}\right)=0$, that is, $x_{i} \in I$ for all $i$, and hence $m \in \sum I f_{i} \subset M^{\prime}$.

Let $P^{\Phi}, S^{\Phi}, T^{\Phi}, U^{\Phi}$ and $V^{\Phi}$ be the algebraic tori over $F$ with the character $G$-modules $R^{r}, Q, M, I$ and $N$, respectively. The diagram of homomorphisms of $G$-modules with exact columns and rows

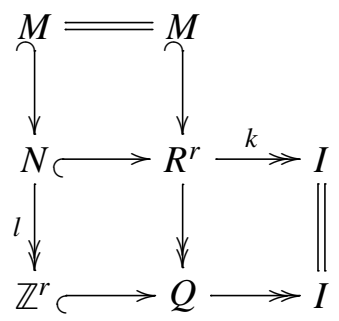

yields the following diagram of homomorphisms of the tori:

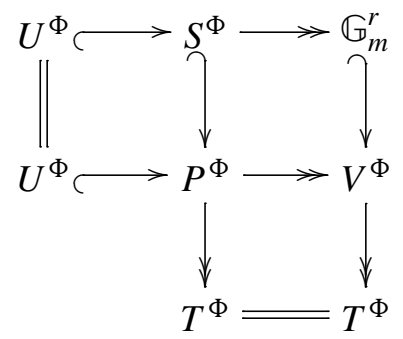

Let $K / F$ be a field extension. Set $K L:=K \otimes_{F} L$. The exact sequence of $G$-modules

$$
0 \rightarrow I \rightarrow R \rightarrow \mathbb{Z} \rightarrow 0
$$

gives an exact sequence of the tori

$$
1 \rightarrow \mathbb{G}_{m} \rightarrow R_{L / F}\left(\mathbb{G}_{m, L}\right) \rightarrow U \rightarrow 1,
$$

and then an exact sequence

$$
0 \rightarrow H^{1}\left(K, U^{\Phi}\right) \rightarrow H^{2}\left(K, \mathbb{G}_{m}\right) \rightarrow H^{2}\left(K L, \mathbb{G}_{m}\right) .
$$

Hence

$$
H^{1}\left(K, U^{\Phi}\right) \simeq \operatorname{Br}(K L / K)
$$

Lemma 3.6. The homomorphism $\left(K^{\times}\right)^{r} \rightarrow H^{1}\left(K, U^{\Phi}\right) \simeq \operatorname{Br}(K L / K)$ induced by the first row of the diagram (3-2) takes $\left(x_{1}, \ldots, x_{r}\right)$ to $\sum_{i=1}^{r}\left(\left(\chi_{i}\right)_{K} \cup\left(x_{i}\right)\right)$. 
Proof. Consider the composition

$$
h: \operatorname{Hom}_{G}\left(\mathbb{Z}^{r}, \mathbb{Z}\right) \rightarrow \operatorname{Ext}_{G}^{1}(I, \mathbb{Z}) \rightarrow \operatorname{Ext}_{G}^{2}(\mathbb{Z}, \mathbb{Z})=H^{2}(G, \mathbb{Z})=\mathrm{Ch}(G),
$$

where the first homomorphism is induced by the bottom row of the diagram (3-1), and the second one by the exact sequence (3-3).

We claim that for any $k$, the image of the $k$ th projection $p_{k}: \mathbb{Z}^{r} \rightarrow \mathbb{Z}$ under the composition (3-5) coincides with $\chi_{k}$. Consider the $G$-homomorphism $R^{r} \rightarrow \mathbb{Q}$, taking $e_{k}$ to $1 / p$ and $e_{i}$ to 0 for all $i \neq k$. By Lemma 3.5, this homomorphism vanishes on $M$, and hence it factors through a map $Q \rightarrow \mathbb{Q}$. Thus, we have a commutative diagram

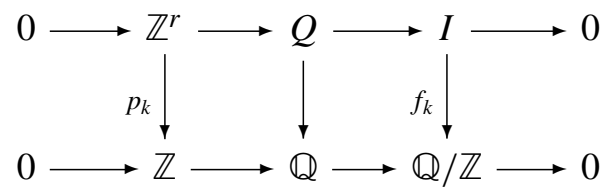

for the map $f_{k}$ defined by $f_{k}\left(\sigma_{k}-1\right)=1 / p+\mathbb{Z}$ and $f_{k}\left(\sigma_{i}-1\right)=0$ for all $i \neq k$.

Let $\alpha$ be the image of the class of the top row of (3-6) under the map $p_{k}^{*}$ : $\operatorname{Ext}_{G}^{1}\left(I, \mathbb{Z}^{r}\right) \rightarrow \operatorname{Ext}_{G}^{1}(I, \mathbb{Z})$. Then $h\left(p_{k}\right)$ is the image of $\alpha$ under the second map in the composition (3-5). Hence $h\left(p_{k}\right)$ is also the image of the class $\beta$ of the sequence (3-3) under the connecting map

$$
H^{1}(G, I)=\operatorname{Ext}_{G}^{1}(\mathbb{Z}, I) \rightarrow \operatorname{Ext}_{G}^{2}(\mathbb{Z}, \mathbb{Z})=H^{2}(G, \mathbb{Z})
$$

induced by the exact sequence representing the class $\alpha$.

The diagram (3-6) yields a commutative diagram

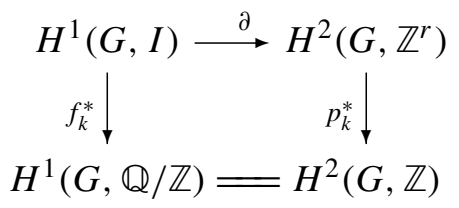

As we have shown, $p_{k}^{*}(\partial(\beta))=h\left(p_{k}\right)$. Therefore, it suffices to prove that $f_{k}^{*}(\beta)=$ $\chi_{k}$. The cocycle $\beta$ satisfies $\beta\left(\sigma_{i}\right)=\sigma_{i}-1$. It follows that $f_{k}^{*}(\beta)\left(\sigma_{k}\right)=f_{k}\left(\sigma_{k}-1\right)=$ $1 / p+\mathbb{Z}$ and $f_{k}^{*}(\beta)\left(\sigma_{i}\right)=0$ for all $i \neq k$. This proves the claim.

Consider the commutative diagram

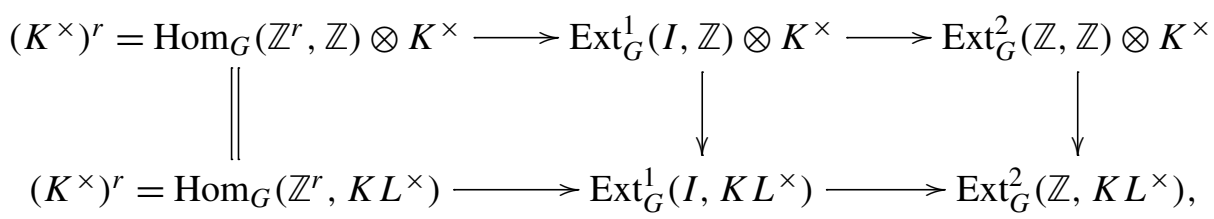

where the vertical homomorphisms are given by the cup products. By the claim, the image of the tuple $\left(x_{1}, \ldots, x_{r}\right)$ under the diagonal composition is equal to 
$\sum_{i=1}^{r}\left(\left(\chi_{i}\right)_{K} \cup\left(x_{i}\right)\right)$. On the other hand, the bottom composition coincides with

$$
\left(K^{\times}\right)^{r} \rightarrow H^{1}\left(K, U^{\Phi}\right) \simeq \operatorname{Br}(K L / K) .
$$

Corollary 3.7. The map $H^{1}\left(K, U^{\Phi}\right) \rightarrow H^{1}\left(K, S^{\Phi}\right)$ induces an isomorphism

$$
H^{1}\left(K, S^{\Phi}\right) \simeq \mathrm{Br}_{\text {ind }}(K L / K)
$$

It follows from Corollary 3.7 and the triviality of the group $H^{1}\left(K, P^{\Phi}\right)$ that we have a commutative diagram

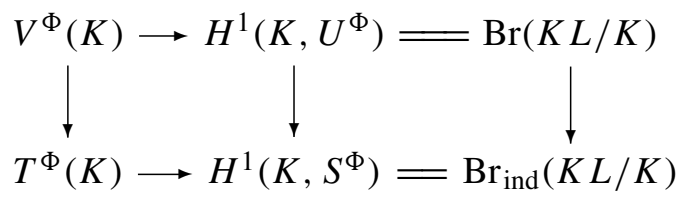

with surjective homomorphisms.

3.1. The element a. Let $a^{\prime}$ be the image of the generic point of $V^{\Phi}$ over $K=$ $F\left(V^{\Phi}\right)$ in $\operatorname{Br}\left(L\left(V^{\Phi}\right) / F\left(V^{\Phi}\right)\right)$ in the diagram (3-7). Choose also an element $a \in$ $\operatorname{Br}\left(L\left(T^{\Phi}\right) / F\left(T^{\Phi}\right)\right)$ corresponding to the generic point of $T^{\Phi}$ over $F\left(T^{\Phi}\right)$. The field $F\left(T^{\Phi}\right)$ is a subfield of $F\left(V^{\Phi}\right)$ and the classes $a_{F\left(V^{\Phi}\right)}$ and $a^{\prime}$ are equal in $\operatorname{Br}_{\text {ind }}\left(L\left(V^{\Phi}\right) / F\left(V^{\Phi}\right)\right)$. It follows that $p a_{F\left(V^{\Phi}\right)}=p a^{\prime}$ in $\operatorname{Br} F\left(V^{\Phi}\right)$.

The exact sequence of $G$-modules

$$
0 \rightarrow L^{\times} \oplus N \rightarrow L\left(V^{\Phi}\right)^{\times} \rightarrow \operatorname{Div}\left(V_{L}^{\Phi}\right) \rightarrow 0
$$

induces an exact sequence

$$
H^{1}\left(G, \operatorname{Div}\left(V_{L}^{\Phi}\right)\right) \rightarrow H^{2}\left(G, L^{\times}\right) \oplus H^{2}(G, N) \rightarrow H^{2}\left(G, L\left(V^{\Phi}\right)^{\times}\right) .
$$

Since $\operatorname{Div}\left(V_{L}^{\Phi}\right)$ is a permutation $G$-module, the first term in the sequence is trivial. Therefore, we get an injective homomorphism

$$
\varphi: H^{2}(G, N) \rightarrow \operatorname{Br} F\left(V^{\Phi}\right) / \operatorname{Br}(F) .
$$

Then (3-1) and (3-3) yield

$$
H^{2}(G, N) \simeq H^{1}(G, I) \simeq \hat{H}^{0}(G, \mathbb{Z})=\mathbb{Z} / p^{r} \mathbb{Z} ;
$$

thus, $H^{2}(G, N)$ has a canonical generator $\xi$ of order $p^{r}$.

Lemma 3.8 [Merkurjev 2010, Lemma 2.4]. We have $\varphi(\xi)=-a^{\prime}+\operatorname{Br}(F)$. 
Proof. Consider the diagram

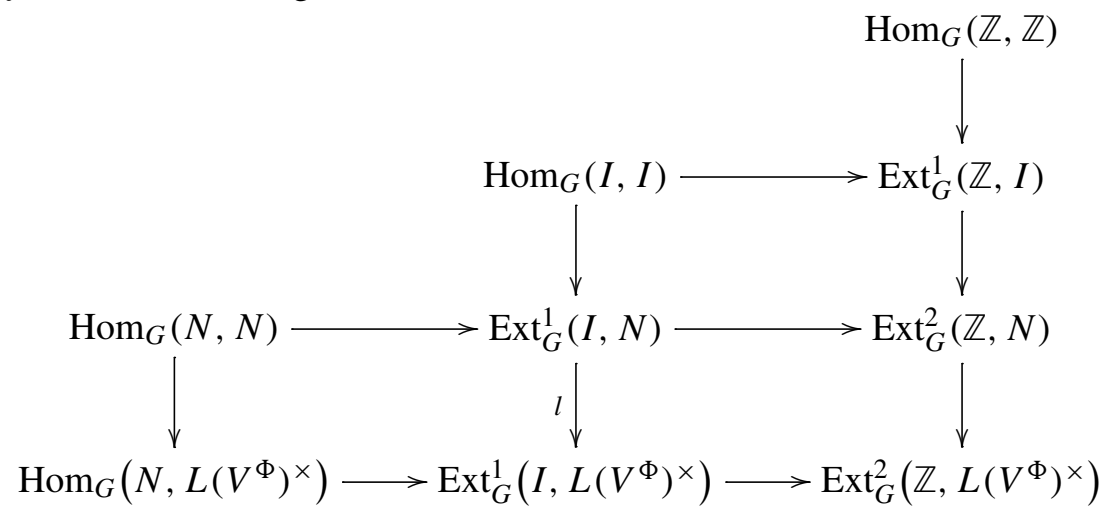

By [Cartan and Eilenberg 1999, Chapter XIV], the images of $1_{\mathbb{Z}}$ and $-1_{I}$ agree in $\operatorname{Ext}_{G}^{1}(\mathbb{Z}, I)$, and the images of $1_{N}$ and $-1_{I}$ agree in $\operatorname{Ext}_{G}^{1}(I, N)$. It follows from [Cartan and Eilenberg 1999, Chapter V, Proposition 4.1] that the upper square is anticommutative. The image of $1_{\mathbb{Z}}$ is equal to $\varphi(\xi)$, and the image of $1_{N}$ is equal to $a^{\prime}+\operatorname{Br}(F)$ in the right bottom corner.

Corollary 3.9. If $r \geq 2$, then the class $p^{r-1} a$ in $\mathrm{Br} F\left(T^{\Phi}\right)$ does not belong to the image of $\operatorname{Br}(F) \rightarrow \operatorname{Br} F\left(T^{\Phi}\right)$.

Proof. The image of $p^{r-1} a$ in $\operatorname{Br} F\left(V^{\Phi}\right)$ coincides with $p^{r-1} a^{\prime}$. Modulo the image of the map $\operatorname{Br}(F) \rightarrow \operatorname{Br} F\left(V^{\Phi}\right)$, the class $p^{r-1} a^{\prime}$ is equal to $-\varphi\left(p^{r-1} \xi\right)$ and is therefore nonzero, since $\varphi$ is injective.

\section{Essential dimension of algebraic tori}

Let $S$ be an algebraic torus over $F$ with the splitting group $G$. We assume that $G$ is a $p$-group of order $p^{r}$. Let $X$ be the $G$-module of characters of $S$. A p-presentation of $X$ is a $G$-homomorphism $f: P \rightarrow X$ with $P$ a permutation $G$-module and finite cokernel of order prime to $p$. A $p$-presentation with the smallest $\operatorname{rank}(P)$ is called minimal.

Essential $p$-dimension of algebraic tori was determined in [Lötscher et al. 2009, Theorem 1.4]:

Theorem 4.1. Let $S$ be an algebraic torus over $F$ with the (finite) splitting group $G, X$ the $G$-module of characters of $S$, and $f: P \rightarrow X$ a minimal p-presentation of $X$. Then $\operatorname{ed}_{p}(S)=\operatorname{rank}(\operatorname{Ker}(f))$.

Corollary 4.2. Suppose that $X$ admits a surjective minimal p-presentation $f$ : $P \rightarrow X$. Then $\operatorname{ed}(S)=\operatorname{ed}_{p}(S)=\operatorname{rank}(\operatorname{Ker}(f))$.

Proof. As explained in Example 3.3, a surjective $G$-homomorphism $f$ yields a generically free representation of $S$ of dimension $\operatorname{rank}(P)$. In view of Section 3 of 
[Reichstein 2000], we have

$$
\operatorname{ed}_{p}(S) \leq \operatorname{ed}(S) \leq \operatorname{rank}(P)-\operatorname{dim}(S)=\operatorname{rank}(\operatorname{Ker}(f)) .
$$

In this section we derive from Theorem 4.1 an explicit formula for the essential $p$-dimension of algebraic tori.

Define the group $\bar{X}:=X /(p X+I X)$, where $I$ is the augmentation ideal in $R=\mathbb{Z}[G]$. For any subgroup $H \subset G$, consider the composition $X^{H} \hookrightarrow X \rightarrow \bar{X}$. For every $k$, let $V_{k}$ denote the image of the homomorphism

$$
\coprod_{H \subset G} X^{H} \rightarrow \bar{X}
$$

where the coproduct is taken over all subgroups $H$ with $[G: H] \leq p^{k}$. We have the sequence of subgroups

$$
0=V_{-1} \subset V_{0} \subset \cdots \subset V_{r}=\bar{X} .
$$

Theorem 4.3. The essential p-dimension of $S$ is given by the explicit formula

$$
\operatorname{ed}_{p}(S)=\sum_{k=0}^{r}\left(\operatorname{rank} V_{k}-\operatorname{rank} V_{k-1}\right) p^{k}-\operatorname{dim}(S) \text {. }
$$

Proof. Set $b_{k}=\operatorname{rank}\left(V_{k}\right)$. By Theorem 4.1, it suffices to prove that the smallest rank of the $G$-module $P$ in a $p$-presentation of $X$ is equal to $\sum_{k=0}^{r}\left(b_{k}-b_{k-1}\right) p^{k}$.

Let $f: P \rightarrow X$ be a $p$-presentation of $X$ and $A$ a $G$-invariant basis of $P$. The set $A$ is the disjoint union of the $G$-orbits $A_{j}$, so that $P$ is the direct sum of the permutation $G$-modules $\mathbb{Z}\left[A_{j}\right]$.

The composition $\bar{f}: P \rightarrow X \rightarrow \bar{X}$ is surjective. Since $G$ acts trivially on $\bar{X}$, the rank of the group $\bar{f}\left(\mathbb{Z}\left[A_{j}\right]\right)$ is at most 1 for all $j$ and $\bar{f}\left(\mathbb{Z}\left[A_{j}\right]\right) \subset V_{k}$ if $\left|A_{j}\right| \leq p^{k}$. It follows that the group $\bar{X} / V_{k}$ is generated by the images under the composition

$$
P \stackrel{\bar{f}}{\rightarrow} \bar{X} \rightarrow \bar{X} / V_{k}
$$

of all $\mathbb{Z}\left[A_{j}\right]$ with $\left|A_{j}\right|>p^{k}$. Denote by $c_{k}$ the number of such orbits $A_{j}$, so that

$$
c_{k} \geq \operatorname{rank}\left(\bar{X} / V_{k}\right)=b_{r}-b_{k} .
$$

Set $c_{k}^{\prime}=b_{r}-c_{k}$, so that $b_{k} \geq c_{k}^{\prime}$ for all $k$ and $b_{r}=c_{r}^{\prime}$.

Since the number of orbits $A_{j}$ with $\left|A_{j}\right|=p^{k}$ is equal to $c_{k-1}-c_{k}$, we have

$$
\begin{aligned}
\operatorname{rank}(P) & =\sum_{k=0}^{r}\left(c_{k-1}-c_{k}\right) p^{k}=\sum_{k=0}^{r}\left(c_{k}^{\prime}-c_{k-1}^{\prime}\right) p^{k}=c_{r}^{\prime} p^{r}+\sum_{k=0}^{r-1} c_{k}^{\prime}\left(p^{k}-p^{k+1}\right) \\
& \geq b_{r} p^{r}+\sum_{k=0}^{r-1} b_{k}\left(p^{k}-p^{k+1}\right)=\sum_{k=0}^{r}\left(b_{k}-b_{k-1}\right) p^{k}
\end{aligned}
$$


It remains to construct a $p$-presentation with $P$ of rank $\sum_{k=0}^{r}\left(b_{k}-b_{k-1}\right) p^{k}$. For every $k \geq 0$, choose a subset $X_{k}$ in $X$ of the preimage of $V_{k}$ under the canonical map $X \rightarrow \bar{X}$, with the property that for any $x \in X_{k}$ there is a subgroup $H_{x} \subset G$ with $x \in X^{H_{x}}$, and $\left[G: H_{x}\right]=p^{k}$ such that the composition

$$
X_{k} \rightarrow V_{k} \rightarrow V_{k} / V_{k-1}
$$

yields a bijection between $X_{k}$ and a basis of $V_{k} / V_{k-1}$. In particular, $\left|X_{k}\right|=$ $b_{k}-b_{k-1}$. Consider the $G$-homomorphism

$$
f: P:=\coprod_{k=0}^{r} \coprod_{x \in X_{k}} \mathbb{Z}\left[G / H_{x}\right] \rightarrow X,
$$

taking 1 in $\mathbb{Z}\left[G / H_{x}\right]$ to $x$ in $X$.

By construction, the composition of $f$ with the canonical map $X \rightarrow \bar{X}$ is surjective. Since $G$ is a $p$-group, the ideal $p R_{(p)}+I$ of $R_{(p)}$ is the Jacobson radical of the ring $R_{(p)}:=R \otimes \mathbb{Z}_{(p)}$. By the Nakayama Lemma, $f_{(p)}$ is surjective. Hence the cokernel of $f$ is finite of order prime to $p$. The rank of the permutation $G$-module $P$ is equal to

$$
\sum_{k=0}^{r} \sum_{x \in X_{k}} p^{k}=\sum_{k=0}^{r}\left|X_{k}\right| p^{k}=\sum_{k=0}^{r}\left(b_{k}-b_{k-1}\right) p^{k} .
$$

Remark 4.4. In the context of finite $p$-groups, Theorem 4.3 was proved in [Meyer and Reichstein 2010, Theorem 1.2].

Example 4.5. Let $F$ be a field and $\Phi$ be a subgroup of ${ }_{p} \mathrm{Ch}(F)$ of rank $r$, and let $L=F(\Phi)$ and $G=\operatorname{Gal}(L / F)$. Consider the torus $U^{\Phi}$ with the character group the augmentation ideal $I$ defined in Section 3.

The middle row of (3-1) yields an exact sequence

$$
\bar{N} \rightarrow(\bar{R})^{r} \rightarrow \bar{I} \rightarrow 0 .
$$

It follows from Lemma 3.4 that $N \subset p R^{r}+I^{r}$, and hence the first homomorphism in the sequence is trivial. The middle group is isomorphic to $(\mathbb{Z} / p \mathbb{Z})^{r}$, and hence $\operatorname{rank}(\bar{I})=r$.

For any subgroup $H \subset G$, the Tate cohomology group $\hat{H}^{0}(H, I) \simeq \hat{H}^{-1}(H, \mathbb{Z})$ is trivial. It follows that the group $I^{H}$ is generated by $N_{H} x$ for all $x \in I$, where $N_{H}=\sum_{h \in H} h \in R$. Since $\bar{I}$ is of period $p$ with trivial $G$-action, the classes of the elements $N_{H} x$ in $\bar{I}$ are trivial if $H$ is a nontrivial subgroup of $G$. It follows that the maps $I^{H} \rightarrow \bar{I}$ are trivial for all $H \neq 1$. In the notation of (4-1), $V_{0}=\cdots=V_{r-1}=0$ and $V_{r}=\bar{I}$. By Theorem 4.3,

$$
\operatorname{ed}_{p}\left(U^{\Phi}\right)=r p^{r}-\operatorname{dim}\left(U^{\Phi}\right)=r p^{r}-p^{r}+1=(r-1) p^{r}+1
$$


and the rank of the permutation module in a minimal $p$-presentation of $I$ is equal to $r p^{r}$. Therefore, $k: R^{r} \rightarrow I$ is a minimal $p$-presentation of $I$ that appears to be surjective. Therefore, by Corollary 4.2,

$$
\operatorname{ed}\left(U^{\Phi}\right)=\operatorname{ed}_{p}\left(U^{\Phi}\right)=(r-1) p^{r}+1 .
$$

Let $S^{\Phi}$ be the torus with the character group $Q$ defined in Section 3. As in (3-1), the homomorphism $k$ factors through a surjective map $R^{r} \rightarrow Q$ that is then necessarily a minimal $p$-presentation of $Q$. By Theorem 4.3 and Corollary 4.2,

$$
\operatorname{ed}\left(S^{\Phi}\right)=\operatorname{ed}_{p}\left(S^{\Phi}\right)=r p^{r}-\operatorname{dim}\left(S^{\Phi}\right)=(r-1) p^{r}-r+1 .
$$

\section{Degeneration}

In this section we study the behavior of the essential $p$-dimension under degeneration, that is, we compare the essential $p$-dimension of an object over a complete discrete valued field and its specialization over the residue field (Proposition 5.2). The iterated degeneration (Corollary 5.4) connects a class in the Brauer group degree $p^{r}$ over some (large) field and the elements of the indecomposable relative Brauer group that are torsors for a certain torus.

A simple degeneration. Let $F$ be a field, $p$ a prime integer different from $\operatorname{char}(F)$, and $\Phi \subset{ }_{p} \mathrm{Ch}(F)$ a finite subgroup. For an integer $k \geq 0$ and a field extension $K / F$, let

$$
\mathscr{B}_{k}^{\Phi}(K)=\left\{a \in \operatorname{Br}(K)\{p\} \text { such that ind } a_{K(\Phi)} \leq p^{k}\right\} .
$$

Two elements $a$ and $a^{\prime}$ in $\mathscr{B}_{k}^{\Phi}(K)$ are equivalent if $a-a^{\prime} \in \operatorname{Br}_{\operatorname{dec}}(K(\Phi) / K)$. Write $\mathscr{F}_{k}^{\Phi}(K)$ for the set of equivalence classes in $\mathscr{B}_{k}^{\Phi}(K)$. Abusing notation, we shall write $a$ for the equivalence class of an element $a \in \mathscr{B}_{k}^{\Phi}(K)$ in $\mathscr{F}_{k}^{\Phi}(K)$.

We view $\mathscr{B}_{k}^{\Phi}$ and $\mathscr{F}_{k}^{\Phi}$ as functors from Fields $/ F$ to Sets.

Example 5.1. (i) If $\Phi$ is the zero subgroup, then $\mathscr{F}_{r}^{\Phi}=\mathscr{B}_{r}^{\Phi} \simeq C S A\left(p^{r}\right) \simeq$ $\operatorname{PGL}\left(p^{r}\right)$ - torsors.

(ii) The set $\mathscr{B}_{0}^{\Phi}(K)$ is naturally bijective to $\operatorname{Br}(K(\Phi) / K)$ and

$$
\mathscr{F}_{0}^{\Phi}(K) \simeq \mathrm{Br}_{\text {ind }}(K(\Phi) / K)
$$

By Corollary 3.7, the latter group is naturally isomorphic to $H^{1}\left(K, S^{\Phi}\right)$, where $S^{\Phi}$ is the torus defined in Section 3, and thus, $\mathscr{F}_{0}^{\Phi} \simeq S^{\Phi}$-torsors.

Let $\Phi^{\prime} \subset \Phi$ be a subgroup of index $p$ and $\eta \in \Phi \backslash \Phi^{\prime}$; hence $\Phi=\left\langle\Phi^{\prime}, \eta\right\rangle$. Let $E / F$ be a field extension such that $\eta_{E} \notin \Phi_{E}^{\prime}$ in $\mathrm{Ch}(E)$. Choose an element $a \in \mathscr{B}_{k}^{\Phi}(E)$, that is, $a \in \operatorname{Br}(E)\{p\}$ and $\operatorname{ind}\left(a_{E(\Phi)}\right) \leq p^{k}$. 
Let $E^{\prime}$ be a field extension of $F$ that is complete with respect to a discrete valuation $v^{\prime}$ over $F$ with residue field $E$, and set

$$
a^{\prime}=\hat{a}+\left(\hat{\eta}_{E} \cup(x)\right) \in \operatorname{Br}\left(E^{\prime}\right)
$$

for some $x \in E^{\prime \times}$ such that $v^{\prime}(x)$ is not divisible by $p$. By Proposition 2.2(ii), $\operatorname{ind}\left(a_{E^{\prime}\left(\Phi^{\prime}\right)}^{\prime}\right)=p \cdot \operatorname{ind}\left(a_{E(\Phi)}\right) \leq p^{k+1}$, and hence $a^{\prime} \in \mathscr{B}_{k+1}^{\Phi^{\prime}}\left(E^{\prime}\right)$.

Proposition 5.2. Suppose that for any finite field extension $N / E$ of degree prime to $p$ and any character $\rho \in \mathrm{Ch}(N)$ of order $p^{2}$ such that $p \cdot \rho \in \Phi_{N} \backslash \Phi_{N}^{\prime}$, we have ind $a_{N\left(\Phi^{\prime}, \rho\right)}>p^{k-1}$. Then

$$
\operatorname{ed}_{p}^{\mathscr{F}_{k+1}^{\Phi^{\prime}}}\left(a^{\prime}\right) \geq \operatorname{ed}_{p}^{\mathscr{F}_{k}^{\Phi}}(a)+1
$$

Proof. Let $M / E^{\prime}$ be a finite field extension of degree prime to $p$, let $M_{0} \subset M$ be a subfield over $F$, and let $a_{0}^{\prime} \in \mathscr{B}_{k+1}^{\Phi^{\prime}}\left(M_{0}\right)$ be such that $\left(a_{0}^{\prime}\right)_{M}=a_{M}^{\prime}$ in $\mathscr{F}_{k+1}^{\Phi^{\prime}}$ and

$$
\text { tr. } \operatorname{deg}_{F}\left(M_{0}\right)=\operatorname{ed}_{p}^{\mathscr{F}_{k+1}^{\Phi^{\prime}}}\left(a^{\prime}\right) .
$$

We have

$$
a_{M}^{\prime}-\left(a_{0}^{\prime}\right)_{M} \in \operatorname{Br}_{\mathrm{dec}}\left(M\left(\Phi^{\prime}\right) / M\right) .
$$

It follows from (5-1) that

$$
a_{M}^{\prime}=\hat{a}_{N}+\left(\hat{\eta}_{N} \cup(x)\right)
$$

and $\partial_{v^{\prime}}\left(a^{\prime}\right)=q \cdot \eta_{E}$, where $q=v^{\prime}(x)$ is relatively prime to $p$. We extend the discrete valuation $v^{\prime}$ on $E^{\prime}$ to a (unique) discrete valuation $v$ on $M$. The ramification index $e^{\prime}$ and inertia degree are both prime to $p$. Thus, the residue field $N$ of $v$ is a finite extension of $E$ of degree prime to $p$. By Proposition 2.2(iii),

$$
\partial_{v}\left(a_{M}^{\prime}\right)=e^{\prime} \cdot \partial_{v^{\prime}}\left(a^{\prime}\right)_{N}=e^{\prime} q \cdot \eta_{N} .
$$

Let $v_{0}$ be the restriction of $v$ to $M_{0}$ and $N_{0}$ its residue field. From (5-2), we have

$$
\partial_{v}\left(a_{M}^{\prime}\right)-\partial_{v}\left(\left(a_{0}^{\prime}\right)_{M}\right) \in \Phi_{N}^{\prime} .
$$

Recall that $\eta_{E} \notin \Phi_{E}^{\prime}$. Since $[N: E]$ is not divisible by $p$, it follows that

$$
\eta_{N} \notin \Phi_{N}^{\prime} .
$$

By (5-4), (5-5) and (5-6), $\partial_{v}\left(\left(a_{0}^{\prime}\right)_{M}\right) \neq 0$, that is, $\left(a_{0}^{\prime}\right)_{M}$ is ramified and therefore $v_{0}$ is nontrivial, that is, $v_{0}$ is a discrete valuation on $M_{0}$.

Let $\eta_{0}:=\partial_{v_{0}}\left(a_{0}^{\prime}\right) \in \mathrm{Ch}\left(N_{0}\right)\{p\}$. By Proposition 2.2(iii),

$$
\partial_{v}\left(\left(a_{0}^{\prime}\right)_{M}\right)=e \cdot\left(\eta_{0}\right)_{N},
$$


where $e$ is the ramification index of $M / M_{0}$, and hence $\left(\eta_{0}\right)_{N} \neq 0$. It follows from (5-4), (5-5) and (5-7) that

$$
e^{\prime} q \cdot \eta_{N}-e \cdot\left(\eta_{0}\right)_{N} \in \Phi_{N}^{\prime} .
$$

Since $e^{\prime} q$ is relatively prime to $p$,

$$
\eta_{N} \in\left\langle\Phi_{N}^{\prime},\left(\eta_{0}\right)_{N}\right\rangle \text { in } \mathrm{Ch}(N) .
$$

Let $p^{t}(t \geq 1)$ be the order of $\left(\eta_{0}\right)_{N}$. It follows from (5-6) and (5-8) that $v_{p}(e)=t-1$ and

$$
p^{t-1} \cdot\left(\eta_{0}\right)_{N} \in \Phi_{N} \backslash \Phi_{N}^{\prime} .
$$

Choose a prime element $\pi_{0}$ in $M_{0}$ and write

$$
\left(a_{0}^{\prime}\right)_{\widehat{M}_{0}}=\hat{a}_{0}+\left(\hat{\eta}_{0} \cup\left(\pi_{0}\right)\right)
$$

in $\operatorname{Br}\left(\widehat{M}_{0}\right)$, where $a_{0} \in \operatorname{Br}\left(N_{0}\right)\{p\}$.

Applying the specialization homomorphism $s_{\pi}: \operatorname{Br}(M)\{p\} \rightarrow \operatorname{Br}(N)\{p\}$ (for a prime element $\pi$ in $M$ ) to (5-2), (5-3) and (5-11), using (2-3) and (5-9), we get

$$
a_{N}-\left(a_{0}\right)_{N} \in \operatorname{Br}_{\operatorname{dec}}\left(N\left(\Phi^{\prime}, \eta_{0}\right) / N\right) .
$$

It follows from (5-12) that

$$
a_{N\left(\Phi^{\prime}, \eta_{0}\right)}=\left(a_{0}\right)_{N\left(\Phi^{\prime}, \eta_{0}\right)}
$$

in $\operatorname{Br}\left(N\left(\Phi^{\prime}, \eta_{0}\right)\right)$.

By $(5-11)$,

$$
\left(a_{0}^{\prime}\right)_{\widehat{M}_{0}\left(\Phi^{\prime}\right)}={\widehat{\left(a_{0}\right)}}_{N_{0}\left(\Phi^{\prime}\right)}+\left({\widehat{\left(\eta_{0}\right)}}_{N_{0}\left(\Phi^{\prime}\right)} \cup\left(\pi_{0}\right)\right) .
$$

Since no nontrivial multiple of $\left(\eta_{0}\right)_{N}$ belongs to $\Phi_{N}^{\prime}$, by (5-10), the order of the character $\left(\eta_{0}\right)_{N_{0}\left(\Phi^{\prime}\right)}$ is at least $p^{t}$. It follows from Proposition 2.2(ii) that

$$
\operatorname{ind}\left(a_{0}\right)_{N_{0}\left(\Phi^{\prime}, \eta_{0}\right)}=\operatorname{ind}\left(a_{0}^{\prime}\right)_{\widehat{M}_{0}\left(\Phi^{\prime}\right)} / \operatorname{ord}\left(\eta_{0}\right)_{N_{0}\left(\Phi^{\prime}\right)} \leq p^{k+1} / p^{t}=p^{k-t+1} .
$$

By (5-13) and (5-14),

$$
\operatorname{ind}\left(a_{N\left(\Phi^{\prime}, \eta_{0}\right)}\right) \leq p^{k-t+1} .
$$

Suppose that $t \geq 2$, and consider the character $\rho=p^{t-2} \cdot\left(\eta_{0}\right)_{N}$ of order $p^{2}$ in $\mathrm{Ch}(N)$. We have $p \cdot \rho=p^{t-1}\left(\eta_{0}\right)_{N} \in \Phi_{N} \backslash \Phi_{N}^{\prime}$, by (5-10). Also, the degree of the field extension $N\left(\Phi^{\prime}, \eta_{0}\right) / N\left(\Phi^{\prime}, \rho\right)$ is equal to $p^{t-2}$. Hence, by (5-15),

$$
\operatorname{ind}\left(a_{N\left(\Phi^{\prime}, \rho\right)}\right) \leq \operatorname{ind}\left(a_{N\left(\Phi^{\prime}, \eta_{0}\right)}\right) \cdot p^{t-2} \leq p^{k-t+1} \cdot p^{t-2}=p^{k-1} .
$$

This contradicts the assumption. Therefore, $t=1$, that is, $\operatorname{ord}\left(\eta_{0}\right)_{N}=p$. Then $(e, p)=1$ and it follows from (5-8) that $\left(\eta_{0}\right)_{N} \in\left\langle\Phi_{N}^{\prime}, \eta_{N}\right\rangle$. Moreover,

$$
\left\langle\Phi^{\prime}, \eta_{0}\right\rangle_{N}=\left\langle\Phi^{\prime}, \eta\right\rangle_{N}=\Phi_{N} .
$$


There is a finite subextension $N_{1} / N_{0}$ of $N / N_{0}$ such that $\left\langle\Phi^{\prime}, \eta_{0}\right\rangle_{N_{1}}=\Phi_{N_{1}}$, by Lemma 2.1. Replacing $N_{0}$ by $N_{1}$ and $a_{0}$ by $\left(a_{0}\right)_{N_{1}}$, we may assume that $\left\langle\Phi^{\prime}, \eta_{0}\right\rangle_{N_{0}}=\Phi_{N_{0}}$. In particular, $\eta_{0}$ is of order $p$ in $\operatorname{Ch}\left(N_{0}\right)$.

Since $\operatorname{ind}\left(a_{0}\right)_{N_{0}(\Phi)}=\operatorname{ind}\left(a_{0}\right)_{N_{0}\left(\Phi^{\prime}, \eta_{0}\right)} \leq p^{k}$ by $(5-14)$, we have $a_{0} \in \mathscr{B}_{k}^{\Phi}\left(N_{0}\right)$.

It follows from (5-12) that

$$
a_{N}-\left(a_{0}\right)_{N} \in \mathrm{Br}_{\mathrm{dec}}(N(\Phi) / N) .
$$

Hence the classes of $a_{N}$ and $\left(a_{0}\right)_{N}$ are equal in $\mathscr{F}_{k}^{\Phi}(N)$. The class of $a_{N}$ in $\mathscr{F}_{k}^{\Phi}(N)$ is then defined over $N_{0}$, and therefore

$$
\operatorname{ed}_{p}^{\mathscr{F}_{k+1}^{\Phi^{\prime}}}\left(a^{\prime}\right)=\operatorname{tr} \cdot \operatorname{deg}_{F}\left(M_{0}\right) \geq \operatorname{tr} \cdot \operatorname{deg}_{F}\left(N_{0}\right)+1 \geq \operatorname{ed}_{p}^{\mathscr{F}_{k}^{\Phi}}(a)+1 .
$$

5.1. Multiple degeneration. In this section we assume that the base field $F$ contains a primitive $p^{2}$-th root of unity.

Let $\chi_{1}, \chi_{2}, \ldots, \chi_{r}$ be linearly independent characters in ${ }_{p} \mathrm{Ch}(F)$, and let $\Phi=$ $\left\langle\chi_{1}, \chi_{2}, \ldots, \chi_{r}\right\rangle$. Let $E / F$ be a field extension such that $\operatorname{rank}\left(\Phi_{E}\right)=r$ and let $a \in \operatorname{Br}(E)\{p\}$ be an element that is split by $E(\Phi)$.

Let $E_{0}=E, E_{1}, \ldots, E_{r}$ be field extensions of $F$ such that for any $k=1,2, \ldots, r$, the field $E_{k}$ is complete with respect to a discrete valuation $v_{k}$ over $F$ and $E_{k-1}$ is its residue field. For any $k=1,2, \ldots, r$, choose elements $x_{k} \in E_{k}^{\times}$such that $v_{k}\left(x_{k}\right)$ is not divisible by $p$, and define the elements $a_{k} \in \operatorname{Br}\left(E_{k}\right)\{p\}$ inductively by $a_{0}=a$ and

$$
a_{k}=\widehat{a_{k-1}}+\left(\widehat{\left(\chi_{k}\right)} E_{k-1} \cup\left(x_{k}\right)\right) .
$$

Let $\Phi_{k}$ be the subgroup of $\Phi$ generated by $\chi_{k+1}, \ldots, \chi_{r}$. Thus, $\Phi_{0}=\Phi, \Phi_{r}=0$ and $\operatorname{rank}\left(\Phi_{k}\right)=r-k$. Note that the character $\left(\chi_{k}\right)_{E_{k-1}\left(\Phi_{k}\right)}$ is not trivial. It follows from Proposition 2.2(ii) that

$$
\operatorname{ind}\left(a_{k}\right)_{E_{k}\left(\Phi_{k}\right)}=p \cdot \operatorname{ind}\left(a_{k-1}\right)_{E_{k-1}\left(\Phi_{k-1}\right)}
$$

for any $k=1, \ldots, r$. Since ind $a_{E(\Phi)}=1$, we have $\operatorname{ind}\left(a_{k}\right)_{E_{k}\left(\Phi_{k}\right)}=p^{k}$ for all $k=0,1, \ldots, r$. In particular, $a_{k} \in \mathscr{B}_{k}^{\Phi_{k}}\left(E_{k}\right)$.

The following lemma assures that under a certain restriction on the element $a$, the conditions of Proposition 5.2 are satisfied for the fields $E_{k}$, the groups of characters $\Phi_{k}$, and the elements $a_{k}$.

Lemma 5.3. Suppose that $a_{E(\Psi)} \notin \operatorname{Im}(\operatorname{Br} F(\Psi) \rightarrow \operatorname{Br} E(\Psi))$ for any proper subgroup $\Psi \subset \Phi$. Then for every $k=0,1, \ldots, r-1$, and any finite field extension $N / E_{k}$ of degree prime to $p$ and any character $\rho \in \mathrm{Ch}(N)$ of order $p^{2}$ such that $p \cdot \rho \in\left(\Phi_{k}\right)_{N} \backslash\left(\Phi_{k+1}\right)_{N}$, we have

$$
\operatorname{ind}\left(a_{k}\right)_{N\left(\Phi_{k+1}, \rho\right)}>p^{k-1} .
$$


Proof. Let $k=0,1, \ldots, r-1$ and $N / E_{k}$ be a finite field extension of degree prime to $p$. We construct a new sequence of fields $\tilde{E}_{0}, \tilde{E}_{1}, \ldots, \tilde{E}_{r}$ such that each $\tilde{E}_{i}$ is a finite extension of $E_{i}$ of degree prime to $p$ as follows. We set $\tilde{E}_{j}=N$. The fields $\tilde{E}_{j}$ with $j<k$ are constructed by descending induction on $j$. If we have constructed $\tilde{E}_{j}$ as a finite extension of $E_{j}$ of degree prime to $p$, then we extend the valuation $v_{j}$ to $\tilde{E}_{j}$ and let $\tilde{E}_{j-1}$ be its residue field. The fields $\tilde{E}_{j}$ with $j>k$ are constructed by induction on $j$. If we have constructed $\tilde{E}_{j}$ as a finite extension of $E_{j}$ of degree prime to $p$, then let $\tilde{E}_{j+1}$ be an extension of $E_{j+1}$ of degree $\left[\tilde{E}_{j}: E_{j}\right.$ ] with residue field $\tilde{E}_{j}$.

Replacing $E_{i}$ by $\tilde{E}_{i}$ and $a_{i}$ by $\left(a_{i}\right)_{\tilde{E}_{i}}$, we may assume that $N=E_{k}$. Let $\rho \in$ $\mathrm{Ch}\left(E_{k}\right)$ be a character of order $p^{2}$. We prove the inequality (5-17) by induction on $r$. The case $r=1$ is obvious. Suppose first that $k<r-1$. Consider the fields $F^{\prime}=F\left(\chi_{r}\right), E^{\prime}=E\left(\chi_{r}\right), E_{i}^{\prime}=E_{i}\left(\chi_{r}\right)$, the sequence of characters $\chi_{i}^{\prime}=\left(\chi_{i}\right)_{F^{\prime}}$, and the sequence of elements $a_{i}^{\prime}:=\left(a_{i}\right)_{E_{i}^{\prime}} \in \operatorname{Br}\left(E_{i}^{\prime}\right)$ for $i=0,1, \ldots, r-1$. Let $\Phi^{\prime}=$ $\left\langle\chi_{1}^{\prime}, \chi_{2}^{\prime}, \ldots, \chi_{r-1}^{\prime}\right\rangle$ and let $\Phi_{k}^{\prime}$ be the subgroup of $\Phi^{\prime}$ generated by $\chi_{k+1}^{\prime}, \ldots, \chi_{r-1}^{\prime}$.

Let $\Psi^{\prime} \subset \Phi^{\prime}$ be a proper subgroup. Then $\Psi:=\Psi^{\prime}+\left\langle\chi_{r}\right\rangle$ is a proper subgroup of $\Phi$. Since $F(\Psi)=F^{\prime}\left(\Psi^{\prime}\right)$ and $E(\Psi)=E^{\prime}\left(\Psi^{\prime}\right)$, we have

$$
a_{E^{\prime}\left(\Psi^{\prime}\right)} \notin \operatorname{Im}\left(\operatorname{Br} F^{\prime}\left(\Psi^{\prime}\right) \rightarrow \operatorname{Br} E^{\prime}\left(\Psi^{\prime}\right)\right) .
$$

By induction, the inequality (5-17) holds for the term $a_{k}^{\prime}$ of the new sequence. Since

$$
\left(a_{k}^{\prime}\right)_{E_{k}^{\prime}\left(\Phi_{k+1}^{\prime}, \rho\right)}=\left(a_{k}\right)_{E_{k}\left(\Phi_{k+1}, \rho\right)},
$$

the inequality (5-17) holds for the term $a_{k}$.

Thus we can assume that $k=r-1$.

Case 1. The character $\rho$ is unramified with respect to $v_{r-1}$, that is, $\rho=\hat{\mu}$ for a character $\mu \in \mathrm{Ch}\left(E_{r-2}\right)$ of order $p^{2}$. By Lemma 2.3(i),

$$
\operatorname{ind}\left(a_{r-2}\right)_{E_{r-2}\left(\chi_{r-1}, \mu\right)}=\operatorname{ind}\left(a_{r-1}\right)_{E_{r-1}(\rho)} / p=\operatorname{ind}\left(a_{r-1}\right)_{E_{r-1}\left(\Phi_{r}, \rho\right)} / p \text {. }
$$

Consider the fields $F^{\prime}=F\left(\chi_{r-1}\right), E^{\prime}=E\left(\chi_{r-1}\right), E_{i}^{\prime}=E_{i}\left(\chi_{r-1}\right)$, the new sequence of characters $\chi_{1}, \ldots, \chi_{r-2}, \chi_{r}$ and the elements $a_{i}^{\prime} \in \operatorname{Br}\left(E_{i}^{\prime}\right)$ for $i=0,1, \ldots, r-1$ defined by $a_{i}^{\prime}=\left(a_{i}\right)_{E_{i}^{\prime}}$ for $i \leq r-2$ and $a_{r-1}^{\prime}=\hat{a}_{r-2}+\left(\hat{\chi}_{r} \cup\left(x_{r-1}\right)\right)$ over $E_{r-1}^{\prime}$.

Let $\Phi^{\prime}=\left\langle\chi_{1}, \ldots, \chi_{r-2}, \chi_{r}\right\rangle$ and $\Psi^{\prime} \subset \Phi^{\prime}$ be a proper subgroup. Then $\Psi:=$ $\Psi^{\prime}+\left\langle\chi_{r-1}\right\rangle$ is a proper subgroup of $\Phi$. Since $F(\Psi)=F^{\prime}\left(\Psi^{\prime}\right)$ and $E(\Psi)=E^{\prime}\left(\Psi^{\prime}\right)$, we have $a_{E^{\prime}\left(\Psi^{\prime}\right)} \notin \operatorname{Im}\left(\mathrm{Br} F^{\prime}\left(\Psi^{\prime}\right) \rightarrow \operatorname{Br} E^{\prime}\left(\Psi^{\prime}\right)\right)$. By induction, the inequality (5-17) holds for the term $a_{r-2}^{\prime}$ of the new sequence, the field $N=E_{r-2}^{\prime}$, and the character $\mu_{N}$. Since

$$
\left(a_{r-2}^{\prime}\right)_{E_{r-2}^{\prime}(\mu)}^{\prime}=\left(a_{r-2}\right)_{E_{r-2}\left(x_{r-1}, \mu\right),}
$$

the equality (5-18) shows that (5-17) holds for $a_{r-1}$. 
Case 2. The character $\rho$ is ramified. Note that $p \cdot \rho$ is a nonzero multiple of $\left(\chi_{r}\right)_{E_{r-1}}$. Suppose the inequality (5-17) fails for $a_{r-1}$, that is, we have

$$
\operatorname{ind}\left(a_{r-1}\right)_{E_{r-1}(\rho)} \leq p^{r-2} .
$$

By Lemma 2.3(ii), there exists a unit $u \in E_{r-1}$ such that $E_{r-2}\left(\chi_{r}\right)=E_{r-2}\left(\bar{u}^{1 / p}\right)$ and

$$
\operatorname{ind}\left(a_{r-2}-\left(\chi_{r-1} \cup\left(\bar{u}^{1 / p}\right)\right)\right)_{E_{r-2}\left(\chi_{r}\right)}=\operatorname{ind}\left(a_{r-1}\right)_{E_{r-1}(\rho)} \leq p^{r-2} \text {. }
$$

By descending induction on $j=0,1, \ldots, r-2$, we show that there exist a unit $u_{j}$ in $E_{j+1}$ and a subgroup $\Theta_{j} \subset \Phi$ of rank $r-j-1$ such that $\chi_{r} \in \Theta_{j}$, $\left\langle\chi_{1}, \ldots, \chi_{j}, \chi_{r-1}\right\rangle \cap \Theta_{j}=0, E_{j}\left(\chi_{r}\right)=E_{j}\left(\bar{u}_{j}^{1 / p}\right)$, and

$$
\operatorname{ind}\left(a_{j}-\left(\chi_{r-1} \cup\left(\bar{u}_{j}^{1 / p}\right)\right)\right)_{E_{j}\left(\Theta_{j}\right)} \leq p^{j} .
$$

If $j=r-2$, we set $u_{j}=u$ and $\Theta_{j}=\left\langle\chi_{r}\right\rangle$.

$(j \Rightarrow j-1)$ : The field $E_{j}\left(\bar{u}_{j}^{1 / p}\right)=E_{j}\left(\chi_{r}\right)$ is unramified over $E_{j}$, and hence $v_{j}\left(\bar{u}_{j}\right)$ is divisible by $p$. Modifying $u_{j}$ by a $p^{2}$-th power, we may assume that $\bar{u}_{j}=u_{j-1} x_{j}^{m p}$ for a unit $u_{j-1} \in E_{j}$ and an integer $m$. Then

$$
\left(a_{j}-\left(\chi_{r-1} \cup\left(\bar{u}_{j}^{1 / p}\right)\right)\right)_{E_{j}\left(\Theta_{j}\right)}=\hat{b}+\left(\hat{\eta} \cup\left(x_{j}\right)\right)_{E_{j}\left(\Theta_{j}\right)},
$$

where $\eta=\chi_{j}-m \chi_{r-1}$ and $b=\left(a_{j-1}-\left(\chi_{r-1} \cup\left(\bar{u}_{j-1}^{1 / p}\right)\right)\right)_{E_{j-1}\left(\Theta_{j}\right)}$. Since $\eta$ is not contained in $\Theta_{j}$, the character $\eta_{E_{j-1}\left(\Theta_{j}\right)}$ is not trivial. Set $\Theta_{j-1}=\left\langle\Theta_{j}, \eta\right\rangle$. It follows from Proposition 2.2(ii) that

$$
\operatorname{ind}\left(b_{E_{j-1}\left(\Theta_{j-1}\right)}\right)=\operatorname{ind}\left(a_{j}-\left(\chi_{r-1} \cup\left(\bar{u}_{j}^{1 / p}\right)\right)\right)_{E_{j}\left(\Theta_{j}\right)} / p \leq p^{j-1} .
$$

Applying the inequality (5-19) in the case $j=0$, we get

$$
a_{E\left(\Theta_{0}\right)}=\left(\chi_{r-1} \cup\left(w^{1 / p}\right)\right)_{E\left(\Theta_{0}\right)}
$$

for an element $w \in E^{\times}$such that $E\left(w^{1 / p}\right)=E\left(\chi_{r}\right)$. Since the character $\chi_{r}$ is defined over $F$, we may assume that $w \in F^{\times}$, and therefore

$$
a_{E\left(\Theta_{0}\right)} \in \operatorname{Im}\left(\operatorname{Br} F\left(\Theta_{0}\right) \rightarrow \operatorname{Br} E\left(\Theta_{0}\right)\right) .
$$

The degree of the extension $E\left(\Theta_{0}\right) / E$ is equal to $p^{r-1}$, and hence $\Theta_{0}$ is a proper subgroup of $\Phi$, a contradiction. Thus, we have shown that the inequality (5-17) holds.

By Example 5.1(ii), we can view $a$ as an $S^{\Phi}$-torsor over $E$.

Corollary 5.4. Suppose that $p^{r-1} a \notin \operatorname{Im}(\operatorname{Br}(F) \rightarrow \operatorname{Br}(E))$. Then

$$
\operatorname{ed}_{p}^{C S A\left(p^{r}\right)}\left(a_{r}\right) \geq \operatorname{ed}_{p}^{S^{\Phi} \text {-torsors }}(a)+r .
$$


Proof. By iterated application of Proposition 5.2 and Example 5.1,

$$
\begin{aligned}
\operatorname{ed}_{p}^{C S A\left(p^{r}\right)}\left(a_{r}\right) & =\operatorname{ed}_{p}^{\mathscr{F}_{r}^{\Phi_{r}}}\left(a_{r}\right) \geq \operatorname{ed}_{p}^{\mathscr{F}_{r-1}^{\Phi_{r-1}}}\left(a_{r-1}\right)+1 \geq \ldots \\
& \geq \operatorname{ed}_{p}^{\mathscr{F}_{1}^{\Phi_{1}}}\left(a_{1}\right)+(r-1) \geq \operatorname{ed}_{p}^{\mathscr{F}_{0}^{\Phi_{0}}}\left(a_{0}\right)+r=\operatorname{ed}_{p}^{S^{\Phi} \text {-torsors }}(a)+r .
\end{aligned}
$$

\section{Proof of the main theorem}

Theorem 6.1. Let $F$ be a field and $p$ a prime integer different from $\operatorname{char}(F)$. Then

$$
\operatorname{ed}_{p}\left(\operatorname{CSA}\left(p^{r}\right)\right) \geq(r-1) p^{r}+1
$$

Proof. Since $\operatorname{ed}_{p}\left(C S A\left(p^{r}\right)\right)$ can only go down if we replace the base field $F$ by any field extension [Merkurjev 2009, Proposition 1.5], we can replace $F$ by any field extension. In particular, we may assume that $F$ contains a primitive $p^{2}$-th root of unity and that there is a subgroup $\Phi$ of ${ }_{p} \mathrm{Ch}(F)$ of rank $r$ (replacing $F$ by the field of rational functions in $r$ variables over $F$ ).

Let $T^{\Phi}$ be the algebraic torus constructed in Section 3 for the subgroup $\Phi$. Set $E=F\left(T^{\Phi}\right)$, and let $a \in \operatorname{Br}(E L / E)$ be the element defined in Section 3.1. Let $a_{r} \in \operatorname{Br}\left(E_{r}\right)$ be the element of index $p^{r}$ constructed in Section 5.1. By Corollary 3.9, the class $p^{r-1} a$ in $\operatorname{Br}(E)$ does not belong to the image of $\operatorname{Br}(F) \rightarrow \operatorname{Br}(E)$. It follows from Corollary 5.4 that

$$
\operatorname{ed}_{p}^{C S A\left(p^{r}\right)}\left(a_{r}\right) \geq \operatorname{ed}_{p}^{S^{\Phi} \text {-torsors }}(a)+r .
$$

The $S^{\Phi}$-torsor $a$ is the generic fiber of the versal $S^{\Phi}$-torsor $P^{\Phi} \rightarrow T^{\Phi}$ (see Example 3.3), and hence $a$ is a generic torsor. By [Reichstein and Youssin 2000, §6] or [Merkurjev 2009, Theorem 2.9],

$$
\operatorname{ed}_{p}^{S^{\Phi} \text { - torsors }}(a)=\operatorname{ed}_{p}\left(S^{\Phi}\right)
$$

The essential $p$-dimension of $S^{\Phi}$ was calculated in (4-3):

$$
\operatorname{ed}_{p}\left(S^{\Phi}\right)=(r-1) p^{r}-r+1
$$

Finally, it follows from (6-1), (6-2) and (6-3) that

$$
\operatorname{ed}_{p}\left(C S A\left(p^{r}\right)\right) \geq \operatorname{ed}_{p}^{C S A\left(p^{r}\right)}\left(a_{r}\right) \geq \operatorname{ed}_{p}^{S^{\Phi} \text {-torsors }}(a)+r=(r-1) p^{r}+1 .
$$

\section{Remarks}

Let $K / F$ be a field extension and $G$ an elementary abelian group of order $p^{r}$. Consider the subset $C S A_{K}(G)$ of $C S A_{K}\left(p^{r}\right)$ consisting of all classes admitting a splitting Galois $K$-algebra $E$ with $\operatorname{Gal}(E / K) \simeq G$. Equivalently, $\operatorname{CSA}_{K}(G)$ 
consists of all classes represented by crossed product algebras with the group $G$ [Herstein 1994, §4.4].

Write $\operatorname{Pair}_{K}(G)$ for the set of isomorphism classes of pairs $(a, E)$, where $a \in$ $C A_{K}(G)$ and $E$ is a Galois $G$-algebra splitting $a$.

Finally, fix a Galois field extension $L / F$ with $\operatorname{Gal}(L / F) \simeq G$ and consider the subset $C S A_{K}(L / F)$ of $C S A_{K}(G)$ consisting of all classes split by the extension $K L / K$. Thus, $C S A(L / F)$ is a subfunctor of $\operatorname{CSA}(G)$ and there is the obvious surjective morphism of functors $\operatorname{Pair}(G) \rightarrow C S A(G)$.

Theorem 7.1. Let $F$ be a field, $p$ a prime integer different from $\operatorname{char}(F), G$ an elementary abelian group of order $p^{r}, r \geq 2$, and $L / F$ a Galois field extension with $\operatorname{Gal}(L / F) \simeq G$. Let $\mathscr{F}$ be one of the three functors $\operatorname{CSA}(L / F), C S A(G)$, Pair $(G)$. Then

$$
\operatorname{ed}(\mathscr{F})=\operatorname{ed}_{p}(\mathscr{F})=(r-1) p^{r}+1 .
$$

Proof. The functor $C S A(L / F)$ is isomorphic to $U^{\Phi}$-torsors by (3-4), where $\Phi$ is a subgroup of $\mathrm{Ch}(F)$ such that $L=F(\Phi)$. It follows from (4-2) that

$$
\operatorname{ed}(C S A(L / F))=\operatorname{ed}_{p}(C S A(L / F))=(r-1) p^{r}+1 .
$$

Let $a_{r}$ be the element in $\operatorname{Br}\left(E_{r}\right)$ in the proof of Theorem 6.1. It satisfies

$$
\operatorname{ed}_{p}^{C S A\left(p^{r}\right)}\left(a_{r}\right) \geq(r-1) p^{r}+1 .
$$

By construction, $a_{r} \in \operatorname{CSA}_{E_{r}}(G)$. Since $\operatorname{CSA}(G)$ is a subfunctor of $\operatorname{CSA}\left(p^{r}\right)$, we have

$$
\operatorname{ed}_{p}(C S A(G)) \geq \operatorname{ed}_{p}^{C S A(G)}\left(a_{r}\right) \geq \operatorname{ed}_{p}^{C S A\left(p^{r}\right)}\left(a_{r}\right) \geq(r-1) p^{r}+1 .
$$

The upper bound $\operatorname{ed}(\operatorname{CSA}(G)) \leq(r-1) p^{r}+1$ was proven in [Lorenz et al. 2003, Corollary 310$]$.

The split étale $F$-algebra $E:=\operatorname{Map}(G, F)$ has the natural structure of a Galois $G$-algebra over $F$. The group $G$ acts on the split torus $U:=R_{E / F}\left(\mathbb{G}_{m, E}\right) / \mathbb{G}_{m}$. Let $A$ be the split $F$-algebra $\operatorname{End}_{F}(E)$. The semidirect product $H:=U \rtimes G$ acts naturally on $A$ by $F$-algebra automorphisms. Moreover, by the Skolem-Noether Theorem, $H$ is precisely the automorphism group of the pair $(A, E)$. It follows that the functor $\operatorname{Pair}_{K}(G)$ is isomorphic to $H$-torsors.

The character group of $U$ is $G$-isomorphic to the ideal $I$ in $R=\mathbb{Z}[G]$. By [Meyer and Reichstein 2009a, §3], the $G$-homomorphism $k: R^{r} \rightarrow I$ constructed in Section 3 yields a representation $W$ of the group $H$ of dimension $r p^{r}$. Since $r \geq 2$, by Lemma 3.4, $G$ acts faithfully on the kernel $N$ of $k$. By [Meyer and Reichstein 2009a, Lemma 3.3], the action of $H$ on $W$ is generically free, and hence

$$
\operatorname{ed}(\operatorname{Pair}(G))=\operatorname{ed}(H) \leq \operatorname{dim}(W)-\operatorname{dim}(H)=(r-1) p^{r}+1 .
$$


Since Pair $(G)$ surjects onto $C S A(G)$, we have

$$
\operatorname{ed}(\operatorname{Pair}(G)) \geq \operatorname{ed}_{p}(\operatorname{Pair}(G)) \geq \operatorname{ed}_{p}(C S A(G))=(r-1) p^{r}+1 .
$$

Remark 7.2. The generic $G$-crossed product algebra $D$ constructed in [Amitsur and Saltman 1978] is a generic element for the functor $C S A(G)$ in the sense of [Merkurjev 2009, §2], and hence

$$
\operatorname{ed}(D)=\operatorname{ed}_{p}(D)=(r-1) p^{r}+1
$$

for $r \geq 2$ by Theorem 7.1 .

\section{References}

[Amitsur and Saltman 1978] S. A. Amitsur and D. Saltman, "Generic Abelian crossed products and p-algebras”, J. Algebra 51:1 (1978), 76-87. MR 58 \#10988 Zbl 0391.13001

[Berhuy and Favi 2003] G. Berhuy and G. Favi, "Essential dimension: a functorial point of view (after A. Merkurjev)”, Doc. Math. 8 (2003), 279-330. MR 2004m:11056 Zbl 1101.14324

[Cartan and Eilenberg 1999] H. Cartan and S. Eilenberg, Homological algebra, Princeton University Press, 1999. MR 2000h:18022 Zbl 0933.18001

[Colliot-Thélène and Sansuc 1977] J.-L. Colliot-Thélène and J.-J. Sansuc, "La $R$-équivalence sur les tores", Ann. Sci. École Norm. Sup. (4) 10:2 (1977), 175-229. MR 56 \#8576 Zbl 0356.14007

[Garibaldi et al. 2003] S. Garibaldi, A. Merkurjev, and J.-P. Serre, Cohomological invariants in Galois cohomology, University Lecture Series 28, American Mathematical Society, Providence, RI, 2003. MR 2004f:11034 Zbl 1159.12311

[Herstein 1994] I. N. Herstein, Noncommutative rings, Carus Mathematical Monographs 15, Mathematical Association of America, Washington, DC, 1994. MR 97m:16001 Zbl 0874.16001

[Jacob and Wadsworth 1990] B. Jacob and A. Wadsworth, "Division algebras over Henselian fields", J. Algebra 128:1 (1990), 126-179. MR 91d:12006 Zbl 0692.16011

[Lorenz et al. 2003] M. Lorenz, Z. Reichstein, L. H. Rowen, and D. J. Saltman, "Fields of definition for division algebras", J. London Math. Soc. (2) 68:3 (2003), 651-670. MR 2004j:16022 Zbl 1071.16012

[Lötscher et al. 2009] R. Lötscher, M. MacDonald, A. Meyer, and R. Reichstein, "Essential pdimension of algebraic tori”, preprint, 2009. arXiv 0910.5574v1

[Matsumura 1980] H. Matsumura, Commutative algebra, 2nd ed., Mathematics Lecture Note Series 56, Benjamin/Cummings, Reading, MA, 1980. MR 82i:13003 Zbl 0441.13001

[Merkurjev 2009] A. S. Merkurjev, "Essential dimension”, pp. 299-325 in Quadratic forms: algebra, arithmetic, and geometry, edited by R. Baeza et al., Contemp. Math. 493, Amer. Math. Soc., Providence, RI, 2009. MR 2010i:14014 Zbl 1188.14006

[Merkurjev 2010] A. S. Merkurjev, "Essential $p$-dimension of PGL $\left(p^{2}\right)$ ", J. Amer. Math. Soc. 23 (2010), 693-712. Zbl 05775681

[Meyer and Reichstein 2009a] A. Meyer and Z. Reichstein, "The essential dimension of the normalizer of a maximal torus in the projective linear group", Algebra Number Theory 3:4 (2009), 467-487. MR 2010h:11065 Zbl 05588054

[Meyer and Reichstein 2009b] A. Meyer and Z. Reichstein, "An upper bound on the essential dimension of a central simple algebra", preprint, 2009, Available at www.math.ubc.ca/ reichst/ meyer-reichstein3.pdf. To appear in J. Algebra. 
[Meyer and Reichstein 2010] A. Meyer and Z. Reichstein, "Some consequences of the KarpenkoMerkurjev theorem", Docum. Math. extra vol. (2010), 445-457. Zbl 05827449

[Reichstein 2000] Z. Reichstein, "On the notion of essential dimension for algebraic groups", Transform. Groups 5:3 (2000), 265-304. MR 2001j:20073 Zbl 0981.20033

[Reichstein and Youssin 2000] Z. Reichstein and B. Youssin, "Essential dimensions of algebraic groups and a resolution theorem for G-varieties", Canad. J. Math. 52:5 (2000), 1018-1056. MR 2001k:14088 Zbl 1044.14023

[Serre 1997] J.-P. Serre, Galois cohomology, Springer, Berlin, 1997. MR 98g:12007 Zbl 0902. 12004

[Tignol 1978] J.-P. Tignol, "Sur les classes de similitude de corps à involution de degré 8", C. $R$. Acad. Sci. Paris Sér. A-B 286:20 (1978), A875-A876. MR 58 \#16605 Zbl 0374.16006

Communicated by Raman Parimala

Received 2009-11-27 Revised 2010-03-30 Accepted 2010-05-15

merkurev@math.ucla.edu

Department of Mathematics, University of California, Los Angeles, CA 90095-1555, United States 


\section{Algebra \& Number Theory}

www.jant.org

\section{EDITORS}

\section{MANAGING EDITOR}

Bjorn Poonen

Massachusetts Institute of Technology

Cambridge, USA

\author{
EDITORIAL BOARD CHAIR \\ David Eisenbud \\ University of California \\ Berkeley, USA
}

\section{BOARD OF EDITORS}

\section{Georgia Benkart}

Dave Benson

Richard E. Borcherds

John H. Coates

J-L. Colliot-Thélène

Brian D. Conrad

Hélène Esnault

Hubert Flenner

Edward Frenkel

Andrew Granville

Joseph Gubeladze

Ehud Hrushovski

Craig Huneke

Mikhail Kapranov

Yujiro Kawamata

János Kollár

Hendrik W. Lenstra

Yuri Manin

Barry Mazur
University of Wisconsin, Madison, USA

University of Aberdeen, Scotland

University of California, Berkeley, USA

University of Cambridge, UK

CNRS, Université Paris-Sud, France

University of Michigan, USA

Universität Duisburg-Essen, Germany

Ruhr-Universität, Germany

University of California, Berkeley, USA

Université de Montréal, Canada

San Francisco State University, USA

Hebrew University, Israel

University of Kansas, USA

Yale University, USA

University of Tokyo, Japan

Princeton University, USA

Universiteit Leiden, The Netherlands

Northwestern University, USA

Harvard University, USA
Susan Montgomery

Shigefumi Mori

Andrei Okounkov

Raman Parimala

Victor Reiner

Karl Rubin

Peter Sarnak

Michael Singer

Ronald Solomon

Vasudevan Srinivas

J. Toby Stafford

Bernd Sturmfels

Richard Taylor

Ravi Vakil

Michel van den Bergh

Marie-France Vignéras

Kei-Ichi Watanabe

Andrei Zelevinsky

Efim Zelmanov
University of Southern California, USA

RIMS, Kyoto University, Japan

Princeton University, USA

Emory University, USA

University of Minnesota, USA

University of California, Irvine, USA

Princeton University, USA

North Carolina State University, USA

Ohio State University, USA

Tata Inst. of Fund. Research, India

University of Michigan, USA

University of California, Berkeley, USA

Harvard University, USA

Stanford University, USA

Hasselt University, Belgium

Université Paris VII, France

Nihon University, Japan

Northeastern University, USA

University of California, San Diego, USA

\section{PRODUCTION}

ant@mathscipub.org

Silvio Levy, Scientific Editor

Andrew Levy, Production Editor

See inside back cover or www.jant.org for submission instructions.

The subscription price for 2010 is US \$140/year for the electronic version, and \$200/year (+\$30 shipping outside the US) for print and electronic. Subscriptions, requests for back issues from the last three years and changes of subscribers address should be sent to Mathematical Sciences Publishers, Department of Mathematics, University of California, Berkeley, CA 94720-3840, USA.

Algebra \& Number Theory (ISSN 1937-0652) at Mathematical Sciences Publishers, Department of Mathematics, University of California, Berkeley, CA 94720-3840 is published continuously online. Periodical rate postage paid at Berkeley, CA 94704, and additional mailing offices.

ANT peer review and production are managed by EditFLOW ${ }^{\mathrm{TM}}$ from Mathematical Sciences Publishers.

\section{PUBLISHED BY}

mathematical sciences publishers

http://www.mathscipub.org

A NON-PROFIT CORPORATION

Typeset in LATEX

Copyright $\odot 2010$ by Mathematical Sciences Publishers 


\section{Algebra \& Number Theory}

Volume $4 \quad$ No. $8 \quad 2010$

On ramification filtrations and $p$-adic differential modules, I: the equal characteristic case

LIANG XIAO

Exponential generation and largeness for compact $p$-adic Lie groups

MICHAEL LARSEN

On the (non)rigidity of the Frobenius endomorphism over Gorenstein rings

HaIlong DaO, JinJia Li and Claudia Miller

A lower bound on the essential dimension of simple algebras

ALEXANDER S. MERKURJEV

On the minimal ramification problem for semiabelian groups

HERShy Kisilevsky, DANNy NEFTIN and JaCK SONN

Remarks on modular symbols for Maass wave forms

YURI I. MANIN 